\title{
Optimal Lipid-Lowering Therapy in Patients Who Were Functionally Deferred Percutaneous Coronary Intervention
}

\author{
Takuro Abe \\ Nishiarai Heart Center Hospital \\ Kensuke Shimazaki \\ Nishiarai Heart Center Hospital \\ Tetsu Moriyama \\ Nishiarai Heart Center Hospital \\ Akira Murohashi \\ Nishiarai Heart Center Hospital \\ Yuji Iwanami \\ Nishiarai Heart Center Hospital \\ Akihito Sasaki \\ Nishiarai Heart Center Hospital \\ Katsumi Saito \\ Nishiarai Heart Center Hospital \\ Kentaro Jujo ( $\sim$ juken1123@mac.com ) \\ Tokyo Women's Medical University
}

\section{Research Article}

Keywords: Fractional flow reserve, Lipid, Percutaneous coronary intervention

Posted Date: February 28th, 2022

DOI: https://doi.org/10.21203/rs.3.rs-1315067/v1

License: (c) (7) This work is licensed under a Creative Commons Attribution 4.0 International License. Read Full License 


\section{Abstract \\ Background}

Deferral of percutaneous coronary intervention (PCl) for functionally insignificant stenosis, defined as fractional flow reserve (FFR) $>0.80$, is associated with favorable long-term prognoses. The lower-the-better strategy for low-density lipoprotein cholesterol (LDL-C) management is an established non-angioplasty therapy to improve the clinical outcomes of patients undergoing PCl. We examined the optimal LDL-C management in cases of intermediate coronary stenosis with deferred $\mathrm{PCl}$ on the basis of FFR values.

\section{Methods}

This observational study included 273 consecutive patients with a single target vessel and deferred PCI with an FFR $>0.80$. Patients with an FFR of $0.81-0.85$ $(n=93)$ and those with FFR $>0.85(n=180)$ were classified into the lower $(<100 \mathrm{mg} / \mathrm{dL})$ and higher $(\geq 100 \mathrm{mg} / \mathrm{dL})$ LDL-C groups. The endpoint was major adverse cardiovascular and cerebrovascular events (MACCE), including death, non-fatal myocardial infarction, ischemic stroke, heart failure hospitalization, and unplanned revascularization.

\section{Results}

Patients with an FFR of $0.81-0.85$ had a significantly higher MACCE rate than those with an FFR $>0.85$ (log-rank, $p=0.003$ ). In patients with an FFR of $0.81-$ 0.85 , the lower LDL-C group showed a significantly lower MACCE rate than the higher LDL-C group (log-rank, $p=0.006)$. However, the event rate did not differ significantly between the two groups in patients with FFR $>0.85$ (log-rank, $p=0.84$ ).

\section{Conclusions}

Uncontrolled LDL-C levels were associated with higher MACCE rates in cases with deferred PCI due to an FFR of $0.81-0.85$. This high-risk population for adverse cardiovascular events should receive strict LDL-C-lowering therapy.

\section{Condensed Abstract}

The residual risk and management in cases with deferred percutaneous coronary intervention (PCl) after fractional flow reserve (FFR) measurement has not been sufficiently evaluated. Our study showed that uncontrolled low-density lipoprotein cholesterol (LDL-C) levels were associated with higher rates of major adverse cardiovascular and cerebrovascular events in cases with deferred PCI due to an FFR of 0.81-0.85. Thus, in cases of deferred PCl, even those involving coronary artery stenosis with relatively lower FFR values should receive strict LDL-C-lowering therapy and close monitoring.

\section{Introduction}

The fractional flow reserve (FFR) is an established diagnostic parameter for identifying myocardial ischemia with coronary artery stenosis. (1) The extent of myocardial ischemia measured using a coronary pressure wire is an important determinant of outcomes in patients with stable coronary artery disease (CAD). FFR has been previously shown to safely indicate whether coronary stenosis requires revascularization by percutaneous coronary intervention (PCl) plus medical therapy or medical therapy alone. (2-5) Current guidelines recommend FFR measurement before revascularization in patients with chronic coronary syndrome without evidence of ischemia from non-invasive testing. (6)

A number of large clinical trials and meta-analyses have established the "the lower, the better" strategy for low-density lipoprotein cholesterol (LDL-C) management in patients after PCl. (7-9) However, the clinical influence of lipid-lowering therapy after deferral of PCl in patients with stenotic lesions has not been well evaluated. Therefore, in clinical practice, patients with angiographically intermediate coronary artery stenosis often receive insufficient lipid-lowering therapy. We aimed to stratify the risk of adverse clinical events in cases where PCl was deferred on the basis of FFR values, focusing on the LDL-C levels during the follow-up period.

\section{Methods}

\section{Study design, population, and endpoints}

This observational study initially included consecutive patients with intermediate coronary artery stenosis who underwent FFR testing at Nishiarai Heart Center Hospital between June 2012 and December 2019. After exclusion of patients with coronary stenosis and FFR $\leq 0.80$, those with multiple stenoses, those without follow-up observations, and those receiving regular hemodialysis, patients with a single target vessel whose PCI had been deferred due to FFR values $>0.80$ were ultimately enrolled in this study. The enrolled patients were divided into two groups depending on the cut-off FFR value of 0.85 , as referred to in a previous report. (5) Patients in each of these FFR-based groups were further classified into two groups based on the LDL-C level at 1 year after the FFR measurement-the lower LDL-C group ( $<100 \mathrm{mg} / \mathrm{dL}$ ) and the higher LDL-C group ( $\geq 100 \mathrm{mg} / \mathrm{dL}$ ) - on the basis of the target LDL-C value for patients who needed secondary prevention of adverse cardiovascular events under the guidelines. $(10,11)$ 
The endpoint of the current study was a major adverse cardiovascular and cerebrovascular event (MACCE), including death, non-fatal myocardial infarction, non-fatal stroke, hospitalization due to worsening of heart failure, and unplanned revascularization after the FFR measurement referred to the previous trials about lipid lowering therapy and FFR. (12) This study complied with the principles of the Declaration of Helsinki. Seiwakai Medical Ethics Committee approved this study protocol and waived the written informed consent because this study was a retrospective observational design.

\section{Coronary angiography and FFR measurement}

Coronary angiography was performed using a guiding catheter via the trans-radial or trans-femoral artery approach with an Allura Xper FD10 (PHILIPS, Amsterdam, the Netherlands) radioscopy system. We conducted FFR measurements in cases of intermediate coronary stenosis estimated to be between $50 \%$ and $90 \%$ by visual inspection without any objective evidence of myocardial ischemia, which was proven by stress myocardial scintigraphy or an exercise stress test. $(6,13)$ The decision to attempt the FFR measurement was made by two or more expert cardiologists in each case. Coronary pressure measurement was performed using a 0.014-inch sensor-tipped guidewire (Pressure Wire: St. Jude Medical, Minnesota, United States; OptoWire: ZEON MEDICAL INC, Tokyo, Japan) introduced through a 5-7-Fr guiding catheter. The pressure wire was calibrated and advanced into the coronary artery and positioned distal to the stenosis as far as possible. Hyperemia was induced by continuous intravenous administration of $150 \mu \mathrm{g} / \mathrm{kg} / \mathrm{min}$ of adenosine. FFR was calculated as the ratio of the mean distal coronary pressure measured by the pressure wire to the mean aortic pressure measured by the guiding catheter during maximal hyperemia.

\section{Data collection and follow-up}

For each patient, we collected data pertaining to the following parameters at the time of FFR measurement: (1) vital signs, (2) medical history, (3) transthoracic echocardiography, (4) quantitative coronary angiography (QCA), and (5) laboratory tests, including hemoglobin, albumin, renal function (creatinine and estimated glomerular filtration rate [eGFR]), B-type natriuretic peptide (BNP), glycated hemoglobin (HbA1C), total cholesterol (T-C), LDL-C, highdensity lipoprotein cholesterol (HDL-C), and triglyceride (TG). Oral medications and laboratory values at the time of FFR measurement and one year after the session were compared among the subgroups. QCA was performed using the Cardiovascular Angiography Analysis System (Pie Medical Imaging). Information about adverse clinical events, including death, non-fatal myocardial infarction, ischemic stroke, hospitalization due to worsening of heart failure, unplanned revascularization, and date of events, was collected by the cardiologist from medical records. The endpoints were analyzed on a per-patient basis. The datasets used and/or analyzed during the current study available from the corresponding author on reasonable request.

\section{Statistical analysis}

The values are expressed as the mean and standard deviation, median and interquartile, and percentiles. The independent Student's t-test and the nonparametric equivalent Mann-Whitney $U$ test were used to compare the groups in relation to the continuous variables, and the chi-squared test and Fischer's exact test were used to compare the groups with respect to the categorical variables, as appropriate. MACCE was evaluated using Kaplan-Meier analysis, and differences between groups were assessed using the log-rank test. Univariate and multivariate analyses were performed using the Cox proportional hazards regression model to evaluate the predictors of MACCE. With respect to the primary endpoint, potential interactions between the FFR (FFR $0.81-0.85$ or FFR > 0.85 ) and LDL-C groups (lower or higher LDL-C groups) were evaluated using logistic regression analysis. Statistical significance was set at a two-sided pvalue of $<0.05$. Statistical analyses were performed using R software, version 3.3.0 (R Foundation for Statistical Computing, Vienna, Austria).

\section{Results}

The current study initially included 571 consecutive lesions that were assessed using the FFR. We excluded 190 cases with FFR $\leq 0.80,39$ cases that required FFR measurements for multiple stenoses, 48 cases with missing LDL-C data at 1 year after the FFR measurement, and 21 cases involving regular hemodialysis. Thus, we finally analyzed 273 lesions in patients with a single target lesion and FFR $>0.80$, including 93 patients with an FFR of $0.81-0.85$ and 180 patients with FFR $>0.85$ (Fig. 1).

Among the 93 patients with an FFR of $0.81-0.85,59$ and 34 were categorized in the lower and higher LDL-C groups, respectively. Among the patients with FFR $>0.85,114$ and 66 were categorized in the lower and higher LDL-C groups, respectively (Fig. 1).

\section{Baseline characteristics}

\section{(i) Entire population}

Table 1 presents the baseline characteristics of the study participants. Overall, the median age was 72 years, and $69 \%$ were men. Compared with patients with FFR $>0.85$, those with an FFR of $0.81-0.85$ had a significantly lower prevalence of hypertension and were subjected to FFR tests significantly more frequently in the left main coronary artery or left anterior descending coronary artery. As for QCA data, there was no significant difference between patients with an FFR of $0.81-0.85$ and those with FFR $>0.85$. Patients with an FFR of $0.81-0.85$ were likely to have lower HDL-C and higher TG values at the time of FFR measurement; however, the two FFR populations showed no significant difference in the lipid profile at 1 year after FFR measurement. In both FFR populations, prescription rates of statins increased from baseline to 1 year after the FFR measurement (FFR, $0.81-0.85$ : $54-61 \%$; FFR > 0.85: 45-62\%), and prescription rates were comparable at all time points in both groups. The statin doses were comparable from FFR up to 1 year after FFR measurement in each group, and the prescription rates of ezetimibe and eicosapentaenoic acid were not significantly different between the FFR measurement and at 1 year after treatment in both groups. None of the patients were using proprotein convertase subtilisin/kexin 9 inhibitors (PCSK9i) at FFR measurement, and only one patient required PCSK9i at 1 year after FFR measurement. 
Table 1

Patient characteristics

\begin{tabular}{|c|c|c|c|c|c|c|c|c|c|}
\hline \multirow[t]{2}{*}{ Variables } & \multicolumn{4}{|c|}{ FFR 0.81-0.85 } & \multicolumn{4}{|l|}{$F F R>0.85$} & \multirow{2}{*}{$\begin{array}{l}\text { FFR } 0.81- \\
0.85 \text { vs. } \\
\text { FFR }>0.85\end{array}$} \\
\hline & \multicolumn{4}{|l|}{$N=93$} & \multicolumn{4}{|l|}{$N=180$} & \\
\hline \multirow[t]{2}{*}{ All } & \multirow{2}{*}{$\begin{array}{l}\text { Lower } \\
\text { LDL-C } \\
\mathbf{n}=\mathbf{5 9}\end{array}$} & \multirow{2}{*}{$\begin{array}{l}\text { Higher } \\
\text { LDL-C }\end{array}$} & \multirow[t]{2}{*}{$P$ value } & \multirow[t]{2}{*}{ All } & $\begin{array}{l}\text { Lower } \\
\text { LDL-C }\end{array}$ & \multicolumn{2}{|c|}{ Higher LDL-C } & \multirow[t]{2}{*}{$\begin{array}{l}P \\
\text { value }\end{array}$} & \\
\hline & & & & & $n=114$ & $n=66$ & & & \\
\hline Age, years & $\begin{array}{l}70.6 \pm \\
10.3\end{array}$ & $\begin{array}{l}71.6 \pm \\
10.1\end{array}$ & $\begin{array}{l}68.9 \pm \\
10.3\end{array}$ & 0.16 & $\begin{array}{l}70.2 \pm \\
10.2\end{array}$ & $71.0 \pm 9.4$ & $68.7 \pm 11.3$ & 0.28 & 0.76 \\
\hline Male & $64(69 \%)$ & $46(78 \%)$ & $18(53 \%)$ & 0.02 & $127(71 \%)$ & $83(73 \%)$ & $44(67 \%)$ & 0.40 & 0.78 \\
\hline $\mathrm{BMI}, \mathrm{kg} / \mathrm{m}^{2}$ & $24.2 \pm 3.2$ & $23.7 \pm 3.2$ & $25.1 \pm 3.1$ & 0.048 & $24.3 \pm 3.6$ & $24.4 \pm 3.3$ & $24.3 \pm 4.2$ & 0.87 & 0.66 \\
\hline Systolic BP, mmHg & $\begin{array}{l}153.1 \pm \\
28.9\end{array}$ & $\begin{array}{l}147.5 \pm \\
25.2\end{array}$ & $\begin{array}{l}162.8 \pm \\
32.1\end{array}$ & 0.03 & $\begin{array}{l}153.6 \pm \\
28.3\end{array}$ & $\begin{array}{l}154.2 \pm \\
28\end{array}$ & $152.6 \pm 28.7$ & 0.45 & 0.64 \\
\hline Diastolic BP, mmHg & $\begin{array}{l}72.3 \pm \\
14.1\end{array}$ & $\begin{array}{l}70.6 \pm \\
12.6\end{array}$ & $75.2 \pm 16$ & 0.21 & $72 \pm 14$ & $\begin{array}{l}71.8 \pm \\
12.2\end{array}$ & $72.2 \pm 16.6$ & 0.71 & 0.92 \\
\hline Heart rate, bpm & $\begin{array}{l}68.9 \pm \\
14.3\end{array}$ & $\begin{array}{l}69.0 \pm \\
16.3\end{array}$ & $\begin{array}{l}68.7 \pm \\
10.0\end{array}$ & 0.69 & $\begin{array}{l}69.8 \pm \\
14.2\end{array}$ & $\begin{array}{l}69.4 \pm \\
14.2\end{array}$ & $70.5 \pm 14.2$ & 0.37 & 0.86 \\
\hline Hypertension & $64(69 \%)$ & $41(69 \%)$ & $23(68 \%)$ & $>0.99$ & $147(82 \%)$ & $93(82 \%)$ & $54(82 \%)$ & $>0.99$ & 0.01 \\
\hline Diabetes & $20(22 \%)$ & $12(20 \%)$ & $8(24 \%)$ & 0.59 & $48(27 \%)$ & $29(25 \%)$ & $19(29 \%)$ & 0.86 & 0.46 \\
\hline Dyslipidemia & $50(54 \%)$ & $31(53 \%)$ & $19(56 \%)$ & 0.83 & $104(58 \%)$ & $70(61 \%)$ & $34(52 \%)$ & 0.2 & 0.37 \\
\hline Smoking & $27(29 \%)$ & $19(32 \%)$ & $8(24 \%)$ & 0.48 & $68(38 \%)$ & 45 (39\%) & $23(35 \%)$ & 0.52 & 0.11 \\
\hline Atrial fibrillation & $5(5 \%)$ & $3(5 \%)$ & $2(6 \%)$ & $>0.99$ & $15(8 \%)$ & $11(10 \%)$ & $4(6 \%)$ & 0.42 & 0.34 \\
\hline Prior MI & $10(11 \%)$ & $9(15 \%)$ & $1(3 \%)$ & 0.09 & $26(14 \%)$ & $18(16 \%)$ & $8(12 \%)$ & 0.51 & 0.45 \\
\hline Prior PCl & $42(45 \%)$ & $28(47 \%)$ & $14(41 \%)$ & 0.67 & $79(44 \%)$ & $56(49 \%)$ & $23(35 \%)$ & 0.09 & 0.90 \\
\hline Prior CABG & $1(1 \%)$ & $0(0 \%)$ & $1(3 \%)$ & 0.37 & $3(2 \%)$ & $2(2 \%)$ & $1(2 \%)$ & $>0.99$ & $>0.99$ \\
\hline PAD & $10(11 \%)$ & $6(10 \%)$ & $4(12 \%)$ & $>0.99$ & $14(8 \%)$ & $11(10 \%)$ & $3(5 \%)$ & 0.26 & 0.50 \\
\hline \multicolumn{10}{|l|}{ Target vessel } \\
\hline LMCA and/or LAD & $75(81 \%)$ & $49(83 \%)$ & $26(76 \%)$ & \multirow[t]{4}{*}{$<0.001$} & $103(57 \%)$ & $68(60 \%)$ & $35(53 \%)$ & \multirow[t]{4}{*}{$<0.001$} & \multirow[t]{4}{*}{$<0.001$} \\
\hline Circumflex & $2(2 \%)$ & $0(0 \%)$ & $2(6 \%)$ & & $20(11 \%)$ & $8(7 \%)$ & $12(18 \%)$ & & \\
\hline $\mathrm{RCA}$ & $15(16 \%)$ & $9(15 \%)$ & $6(18 \%)$ & & $54(30 \%)$ & $36(32 \%)$ & $18(27 \%)$ & & \\
\hline Others & $2(2 \%)$ & $1(2 \%)$ & $1(3 \%)$ & & $3(2 \%)$ & $2(2 \%)$ & $1(2 \%)$ & & \\
\hline \multicolumn{10}{|c|}{ Quantitative coronary angiography } \\
\hline $\begin{array}{l}\text { Reference vessel } \\
\text { diameter }\end{array}$ & $2.6 \pm 0.6$ & $2.7 \pm 0.6$ & $2.6 \pm 0.6$ & 0.66 & $2.8 \pm 0.7$ & $2.8 \pm 0.6$ & $2.7 \pm 0.8$ & 0.58 & 0.25 \\
\hline $\begin{array}{l}\text { Minimal lumen } \\
\text { diameter }\end{array}$ & $1.4 \pm 0.5$ & $1.4 \pm 0.5$ & $1.4 \pm 0.4$ & 0.85 & $1.5 \pm 0.6$ & $1.5 \pm 0.6$ & $1.5 \pm 0.6$ & 0.19 & 0.38 \\
\hline \% diameter stenosis & $\begin{array}{l}46.1 \pm \\
13.9\end{array}$ & $\begin{array}{l}46.6 \pm \\
13.8\end{array}$ & $\begin{array}{l}45.3 \pm \\
14.1\end{array}$ & 0.65 & $\begin{array}{l}46.6 \pm \\
14.3\end{array}$ & $\begin{array}{l}46.1 \pm \\
14.5\end{array}$ & $47.4 \pm 13.8$ & 0.64 & 0.86 \\
\hline Lesion length & $9.7 \pm 5.2$ & $9.9 \pm 4.9$ & $9.3 \pm 5.7$ & 0.35 & $9.7 \pm 5.2$ & $9.8 \pm 5.5$ & $9.5 \pm 4.8$ & 0.90 & 0.99 \\
\hline \multicolumn{10}{|c|}{ Laboratory data at FFR measurement } \\
\hline Hemoglobin, g/dL & $13.6 \pm 1.6$ & $13.7 \pm 1.4$ & $13.4 \pm 2.1$ & 0.64 & $13.9 \pm 1.9$ & $14 \pm 1.8$ & $13.7 \pm 2.0$ & 0.51 & 0.44 \\
\hline $\begin{array}{l}\text { eGFR, } \\
\mathrm{mL} / \mathrm{min} / 1.73 \mathrm{~m}^{2}\end{array}$ & $\begin{array}{l}60.1 \pm \\
22.7\end{array}$ & $\begin{array}{l}58.5 \pm \\
21.1\end{array}$ & $\begin{array}{l}62.9 \pm \\
25.1\end{array}$ & 0.58 & $\begin{array}{l}60.8 \pm \\
25.7\end{array}$ & $\begin{array}{l}59.6 \pm \\
25.9\end{array}$ & $62.9 \pm 25.3$ & 0.42 & 0.83 \\
\hline
\end{tabular}

Abbreviations: $\mathrm{ACEi}=$ angiotensin converting enzyme inhibitors; $\mathrm{ARB}=$ angiotensin II receptor blockers; $\mathrm{BMI}=$ body mass index; $\mathrm{BNP}=$ brain natriuretic peptide; $\mathrm{BP}=$ blood pressure; $\mathrm{CABG}=$ coronary artery bypass grafting; DPP4i = dipeptidyl peptidase- 4 inhibitors; $\mathrm{EPA}=$ eicosapentaenoic acid; FFR = fractional flow reserve; $\mathrm{HDL}-\mathrm{C}=$ high density lipoprotein cholesterol; $\mathrm{HbA} 1 \mathrm{C}=$ Hemoglobin $\mathrm{A} 1 \mathrm{c} ; \mathrm{LAD}=$ left anterior descending coronary artery; $\mathrm{LCX}=$ left circumflex coronary artery; $L / D=$ laboratory data; $L D L-C=$ low-density lipoprotein cholesterol; $L M C A=$ left main coronary artery; $L V E F=$ left ventricular ejection fraction; $\mathrm{MI}=$ myocardial infarction; $\mathrm{MLD}=$ minimum lumen diameter; $\mathrm{MRA}=$ mineralocorticoid receptor antagonists; $\mathrm{PCSK}-9=\mathrm{Proprotein}$ convertase subtilisin/kexin type 9; $\mathrm{PAD}=$ peripheral artery disease; $\mathrm{PCI}=$ percutaneous coronary intervention; \%DS = \% diameter stenosis; $\mathrm{PPI}=\mathrm{Proton}$ pump inhibitors; RCA = right coronary artery; $\mathrm{SGLT} 2 \mathrm{i}=$ sodium glucose cotransporter 2 inhibitors; $\mathrm{T}-\mathrm{C}=$ total cholesterol. 


\begin{tabular}{|c|c|c|c|c|c|c|c|c|c|}
\hline \multirow[t]{2}{*}{ Variables } & \multicolumn{4}{|c|}{ FFR $0.81-0.85$} & \multicolumn{4}{|l|}{ FFR $>0.85$} & \multirow{2}{*}{$\begin{array}{l}\text { FFR } 0.81- \\
0.85 \text { vs. } \\
\text { FFR }>0.85\end{array}$} \\
\hline & \multicolumn{4}{|l|}{$N=93$} & \multicolumn{4}{|l|}{$N=180$} & \\
\hline $\mathrm{T}-\mathrm{C}, \mathrm{mg} / \mathrm{dL}$ & $\begin{array}{l}187.3 \pm \\
54.9\end{array}$ & $\begin{array}{l}177.1 \pm \\
38.3\end{array}$ & $\begin{array}{l}204.2 \pm \\
71.8\end{array}$ & 0.02 & $\begin{array}{l}181 \pm \\
36.2\end{array}$ & $\begin{array}{l}174.8 \pm \\
37.2\end{array}$ & $191.9 \pm 31.7$ & 0.002 & 0.64 \\
\hline LDL-C, mg/dL & $\begin{array}{l}105.5 \pm \\
38.5\end{array}$ & $\begin{array}{l}100.3 \pm \\
38.2\end{array}$ & $\begin{array}{l}114.5 \pm \\
37.4\end{array}$ & 0.06 & $98.5 \pm 31$ & $\begin{array}{l}91.9 \pm \\
31.9\end{array}$ & $110.0 \pm 25.5$ & $<0.001$ & 0.39 \\
\hline $\mathrm{HDL}-\mathrm{C}, \mathrm{mg} / \mathrm{dL}$ & $\begin{array}{l}51.5 \pm \\
18.6\end{array}$ & $\begin{array}{l}53.1 \pm \\
20.9\end{array}$ & $\begin{array}{l}48.8 \pm \\
13.6\end{array}$ & 0.57 & $56.6 \pm 19$ & $\begin{array}{l}57.2 \pm \\
19.5\end{array}$ & $55.6 \pm 18.0$ & 0.88 & 0.01 \\
\hline $\mathrm{TG}, \mathrm{mg} / \mathrm{dL}$ & $\begin{array}{l}167.9 \pm \\
154.9\end{array}$ & $\begin{array}{l}140.4 \pm \\
71.7\end{array}$ & $\begin{array}{l}214.8 \pm \\
229.4\end{array}$ & 0.02 & $\begin{array}{l}138.4 \pm \\
100.7\end{array}$ & $\begin{array}{l}135.6 \pm \\
99\end{array}$ & $143.3 \pm 103.4$ & 0.89 & 0.003 \\
\hline $\mathrm{HbA1c} \%$ & $6.2 \pm 1.1$ & $6.2 \pm 0.9$ & $6.3 \pm 1.2$ & 0.52 & $6.2 \pm 1.2$ & $6.2 \pm 1.3$ & $6.2 \pm 1$ & 0.57 & 0.36 \\
\hline \multicolumn{10}{|c|}{ Laboratory data at 1 year after FFR measurement } \\
\hline Hemoglobin, g/dL & $13.4 \pm 1.7$ & $13.4 \pm 1.6$ & $13.3 \pm 1.9$ & 0.93 & $13.5 \pm 1.9$ & $13.3 \pm 1.8$ & $13.8 \pm 1.9$ & 0.071 & 0.99 \\
\hline $\begin{array}{l}\text { eGFR, } \\
\mathrm{mL} / \mathrm{min} / 1.73 \mathrm{~m}^{2}\end{array}$ & $\begin{array}{l}58.3 \pm \\
21.9\end{array}$ & $\begin{array}{l}57.6 \pm \\
20.4\end{array}$ & $\begin{array}{l}59.5 \pm \\
24.3\end{array}$ & 0.83 & $56 \pm 23.3$ & $\begin{array}{l}55.9 \pm \\
23.5\end{array}$ & $56.1 \pm 22.8$ & 0.90 & 0.23 \\
\hline $\mathrm{T}-\mathrm{C}, \mathrm{mg} / \mathrm{dL}$ & $\begin{array}{l}174.7 \pm \\
44.6\end{array}$ & $\begin{array}{l}153.9 \pm \\
24.2\end{array}$ & $\begin{array}{l}210.2 \pm \\
48.9\end{array}$ & $<0.001$ & $\begin{array}{l}175.5 \pm \\
45.1\end{array}$ & $\begin{array}{l}154.9 \pm \\
27.9\end{array}$ & $209.2 \pm 47.5$ & $<0.001$ & 0.65 \\
\hline LDL-C, mg/dL & $\begin{array}{l}91.4 \pm \\
28.4\end{array}$ & $74.4 \pm 17$ & $\begin{array}{l}120.9 \pm \\
18.4\end{array}$ & $<0.001$ & $\begin{array}{l}94.9 \pm \\
35.5\end{array}$ & $\begin{array}{l}74.1 \pm \\
16.7\end{array}$ & $130.8 \pm 30.4$ & $<0.001$ & 0.77 \\
\hline $\mathrm{HDL}-\mathrm{C}, \mathrm{mg} / \mathrm{dL}$ & $\begin{array}{l}52.6 \pm \\
15.2\end{array}$ & $\begin{array}{l}53.3 \pm \\
15.8\end{array}$ & $\begin{array}{l}51.5 \pm \\
13.9\end{array}$ & 0.74 & $56.1 \pm 18$ & $\begin{array}{l}55.3 \pm \\
17.6\end{array}$ & $57.4 \pm 18.7$ & 0.44 & 0.18 \\
\hline Triglyceride, mg/dL & $\begin{array}{l}174.5 \pm \\
161.7\end{array}$ & $\begin{array}{l}158.1 \pm \\
135.4\end{array}$ & $\begin{array}{l}202.9 \pm \\
196\end{array}$ & 0.12 & $\begin{array}{l}157 \pm \\
131.7\end{array}$ & $\begin{array}{l}156.9 \pm \\
143.2\end{array}$ & $157.1 \pm 108.4$ & 0.52 & 0.50 \\
\hline HbA1c, \% & $6 \pm 0.7$ & $6.0 \pm 0.7$ & $6.0 \pm 0.7$ & 0.78 & $6.5 \pm 6.6$ & $6.8 \pm 8.3$ & $6.2 \pm 1.2$ & 0.85 & 0.54 \\
\hline \multicolumn{10}{|c|}{ Medications at FFR measurement } \\
\hline Aspirin & $55(59 \%)$ & $39(66 \%)$ & $16(47 \%)$ & 0.08 & $83(46 \%)$ & $62(54 \%)$ & $21(32 \%)$ & 0.005 & 0.06 \\
\hline Clopidogrel & $31(33 \%)$ & $21(36 \%)$ & $10(29 \%)$ & 0.65 & $49(27 \%)$ & $32(28 \%)$ & $17(26 \%)$ & 0.86 & 0.33 \\
\hline Prasugrel & $10(11 \%)$ & $6(10 \%)$ & $4(12 \%)$ & $>0.99$ & $22(12 \%)$ & $18(16 \%)$ & $4(6 \%)$ & 0.06 & 0.84 \\
\hline Oral anticoagulant & $7(8 \%)$ & $5(8 \%)$ & $2(6 \%)$ & $>0.99$ & $17(9 \%)$ & $11(10 \%)$ & $6(9 \%)$ & $>0.99$ & 0.66 \\
\hline Beta blocker & 31 (33\%) & $22(37 \%)$ & $9(26 \%)$ & 0.36 & $62(34 \%)$ & $43(38 \%)$ & $19(29 \%)$ & 0.26 & 0.89 \\
\hline ACEi/ARB & 32 (34\%) & 22 (37\%) & $10(29 \%)$ & 0.50 & 69 (38\%) & $47(41 \%)$ & $22(33 \%)$ & 0.34 & 0.60 \\
\hline MRA & $6(6 \%)$ & $4(7 \%)$ & $2(6 \%)$ & $>0.99$ & $13(7 \%)$ & $9(8 \%)$ & $4(6 \%)$ & 0.77 & $>0.99$ \\
\hline $\begin{array}{l}\text { Calcium channel } \\
\text { blocker }\end{array}$ & 31 (33\%) & $21(36 \%)$ & $10(29 \%)$ & 0.65 & $55(31 \%)$ & 35 (31\%) & $20(30 \%)$ & $>0.99$ & 0.68 \\
\hline Statins & $50(54 \%)$ & $40(68 \%)$ & $10(29 \%)$ & $<0.001$ & $81(45 \%)$ & $62(54 \%)$ & $19(29 \%)$ & $<0.001$ & 0.20 \\
\hline Rosuvastatin & $29(31 \%)$ & $24(41 \%)$ & $5(15 \%)$ & & $45(25 \%)$ & $34(30 \%)$ & $11(17 \%)$ & & \\
\hline $\begin{array}{l}\text { Rosuvastatin dose } \\
\text { (mg) }\end{array}$ & $3.7 \pm 1.2$ & $3.6 \pm 1.2$ & $4.0 \pm 1.2$ & & $4.0 \pm 1.8$ & $3.8 \pm 1.3$ & $4.8 \pm 2.7$ & & \\
\hline Atorvastatin & $7(8 \%)$ & $6(10 \%)$ & $1(3 \%)$ & & $14(8 \%)$ & $10(9 \%)$ & $4(6 \%)$ & & \\
\hline $\begin{array}{l}\text { Atorvastatin dose } \\
\text { (mg) }\end{array}$ & $11.4 \pm 3.5$ & $11.7 \pm 3.7$ & $10 \pm 0$ & & $9.3 \pm 4.9$ & $7.5 \pm 2.5$ & $13.8 \pm 6.5$ & & \\
\hline Pitavastatin & $13(25 \%)$ & $9(15 \%)$ & $4(10 \%)$ & & $17(10 \%)$ & $15(13 \%)$ & $2(3 \%)$ & & \\
\hline $\begin{array}{l}\text { Pitavastatin dose } \\
\text { (mg) }\end{array}$ & $2.2 \pm 0.5$ & $2.0 \pm 0$ & $2.5 \pm 0.9$ & & $2.3 \pm 0.7$ & $2.3 \pm 0.7$ & $2.0 \pm 0$ & & \\
\hline Others & $1(1 \%)$ & $1(2 \%)$ & $0(0 \%)$ & & $5(3 \%)$ & $3(3 \%)$ & $2(3 \%)$ & & \\
\hline $\begin{array}{l}\text { Abbreviations: ACEi } \\
\text { peptide; BP = blood p } \\
\text { fractional flow reserv } \\
\text { circumflex coronary } \\
\text { ejection fraction; MI = } \\
\text { convertase subtilisin } \\
\text { pump inhibitors; RCA }\end{array}$ & $\begin{array}{l}\text { ngiotensin c } \\
\text { ssure; } C A B G \\
\mathrm{HDL}-\mathrm{C}=\text { hig } \\
\text { ery; L/D = la } \\
\text { nyocardial ir } \\
\text { exin type } 9 ; \\
\text { right corona }\end{array}$ & $\begin{array}{l}\text { nverting enz } \\
\text { coronary ar } \\
\text { density lipo } \\
\text { ratory data } \\
\text { arction; MLD } \\
A D=\text { periphe } \\
\text { artery; SGL }\end{array}$ & $\begin{array}{l}\text { ne inhibitor } \\
\text { y bypass g } \\
\text { ptein choles } \\
\text { DL-C= low- } \\
\text { minimum I } \\
\text { artery dise } \\
\mathrm{i}=\text { sodium }\end{array}$ & $\begin{array}{l}\text { ARB = anc } \\
\text { fting; DPP } \\
\text { rol; HbA1 } \\
\text { ensity lipo } \\
\text { men diam } \\
\text { se; PCl = p } \\
\text { ucose cot }\end{array}$ & $\begin{array}{l}\text { ensin II rece } \\
\text { dipeptidyl } \\
\text { temoglobin } \\
\text { ein cholest } \\
\text {; MRA = mir } \\
\text { taneous co } \\
\text { sporter } 2 \text { int }\end{array}$ & $\begin{array}{l}\text { or blockers; } \\
\text { ptidase-4 in } \\
1 \mathrm{C} ; \mathrm{LAD}=\mathrm{le} \\
\text { l; LMCA = le } \\
\text { alocorticoic } \\
\text { nary interve } \\
\text { itors; T-C = t }\end{array}$ & $\begin{array}{l}\mathrm{MI}=\text { body mass } \\
\text { bitors; EPA = eic } \\
\text { anterior descen } \\
\text { main coronary } \\
\text { eceptor antagon } \\
\text { ion; \%DS }=\% \text { di } \\
\text { al cholesterol. }\end{array}$ & $\begin{array}{l}\mathrm{VP}=\text { brain } \\
\text { enoic aci } \\
\text { nary arter } \\
\mathrm{EF}=\text { left } \mathrm{V} \\
\mathrm{K}-9=\text { Prop } \\
\text { enosis; } \mathrm{P}\end{array}$ & $\begin{array}{l}\text { latriuretic } \\
\text { FFR = } \\
\text { LCX = left } \\
\text { ntricular } \\
\text { otein } \\
=\text { Proton }\end{array}$ \\
\hline
\end{tabular}




\begin{tabular}{|c|c|c|c|c|c|c|c|c|c|}
\hline \multirow[t]{2}{*}{ Variables } & \multirow{2}{*}{\multicolumn{2}{|c|}{$\begin{array}{l}\text { FFR } 0.81-0.85 \\
N=93\end{array}$}} & & & \multirow{2}{*}{\multicolumn{2}{|c|}{$\begin{array}{l}F F R>0.85 \\
N=180\end{array}$}} & & & \multirow{2}{*}{$\begin{array}{l}\text { FFR } 0.81- \\
0.85 \text { vs. } \\
\text { FFR }>0.85\end{array}$} \\
\hline & & & & & & & & & \\
\hline Ezetimibe & $7(8 \%)$ & $4(7 \%)$ & $3(9 \%)$ & 0.7 & $13(7 \%)$ & $9(8 \%)$ & $4(6 \%)$ & 0.77 & $>0.99$ \\
\hline PCSK-9 inhibitors & $0(0 \%)$ & $0(0 \%)$ & $0(0 \%)$ & N.S & $0(0 \%)$ & $0(0 \%)$ & $0(0 \%)$ & N.S & N.S \\
\hline EPA & $8(9 \%)$ & $6(10 \%)$ & $2(6 \%)$ & 0.71 & $12(7 \%)$ & $10(9 \%)$ & $2(3 \%)$ & 0.22 & 0.63 \\
\hline Metformin & $3(3 \%)$ & $1(2 \%)$ & $2(6 \%)$ & 0.55 & $13(7 \%)$ & $10(9 \%)$ & $3(5 \%)$ & 0.38 & 0.28 \\
\hline DPP4i & $10(11 \%)$ & $6(10 \%)$ & $4(12 \%)$ & $>0.99$ & $19(11 \%)$ & $13(11 \%)$ & $6(9 \%)$ & 0.8 & $>0.99$ \\
\hline SGLT2i & $3(3 \%)$ & $3(5 \%)$ & $0(0 \%)$ & 0.30 & $2(1 \%)$ & $2(2 \%)$ & $0(0 \%)$ & 0.53 & 0.34 \\
\hline PPI & 33 (35\%) & $28(47 \%)$ & $5(15 \%)$ & 0.002 & $48(27 \%)$ & $36(32 \%)$ & $12(18 \%)$ & 0.056 & 0.66 \\
\hline Diuretic & $11(12 \%)$ & $7(12 \%)$ & $4(12 \%)$ & $>0.99$ & $16(9 \%)$ & $12(11 \%)$ & $4(6 \%)$ & 0.42 & 0.52 \\
\hline \multicolumn{10}{|c|}{ Medications at 1 year after FFR measurement } \\
\hline Aspirin & $48(52 \%)$ & $34(58 \%)$ & $14(41 \%)$ & 0.14 & $75(42 \%)$ & $56(49 \%)$ & $19(29 \%)$ & 0.01 & 0.13 \\
\hline Clopidogrel & $28(30 \%)$ & $18(31 \%)$ & $10(29 \%)$ & $>0.99$ & $47(26 \%)$ & $37(32 \%)$ & $10(15 \%)$ & 0.01 & 0.48 \\
\hline Prasugrel & $7(8 \%)$ & $5(8 \%)$ & $2(6 \%)$ & $>0.99$ & $17(9 \%)$ & $11(10 \%)$ & $6(9 \%)$ & $>0.99$ & 0.66 \\
\hline Oral anticoagulant & $7(8 \%)$ & $4(7 \%)$ & $3(9 \%)$ & 0.70 & $20(11 \%)$ & $13(11 \%)$ & $7(11 \%)$ & $>0.99$ & 0.40 \\
\hline Beta blocker & $33(35 \%)$ & $22(37 \%)$ & $11(32 \%)$ & 0.66 & $73(41 \%)$ & $57(50 \%)$ & $16(24 \%)$ & $<0.001$ & 0.44 \\
\hline ACEi/ARB & 37 (40\%) & $28(47 \%)$ & $9(26 \%)$ & 0.052 & 73 (41\%) & $56(49 \%)$ & $17(26 \%)$ & 0.003 & $>0.99$ \\
\hline MRA & $8(9 \%)$ & $6(10 \%)$ & $2(6 \%)$ & 0.71 & $14(8 \%)$ & $7(6 \%)$ & $7(11 \%)$ & 0.39 & 0.82 \\
\hline $\begin{array}{l}\text { Calcium channel } \\
\text { blocker }\end{array}$ & $29(31 \%)$ & 20 (34\%) & $9(26 \%)$ & 0.5 & $48(27 \%)$ & 29 (25\%) & $19(29 \%)$ & 0.73 & 0.48 \\
\hline Statins & $57(61 \%)$ & 45 (76\%) & $12(35 \%)$ & $<0.001$ & $112(62 \%)$ & $86(75 \%)$ & $26(39 \%)$ & $<0.001$ & 0.90 \\
\hline Rosuvastatin & 32 (34\%) & $26(44 \%)$ & $6(18 \%)$ & & 70 (39\%) & $52(46 \%)$ & $18(27 \%)$ & & \\
\hline $\begin{array}{l}\text { Rosuvastatin dose } \\
\text { (mg) }\end{array}$ & $3.8 \pm 1.2$ & $3.9 \pm 1.2$ & $3.3 \pm 1.2$ & & $4.0 \pm 1.8$ & $3.8 \pm 1.2$ & $4.6 \pm 2.7$ & & \\
\hline Atorvastatin & $13(14 \%)$ & 10 (17\%) & $3(9 \%)$ & & $16(9 \%)$ & $11(10 \%)$ & $5(8 \%)$ & & \\
\hline $\begin{array}{l}\text { Atorvastatin dose } \\
\text { (mg) }\end{array}$ & $10.4 \pm 4.6$ & $11.5 \pm 4.5$ & $6.7 \pm 2.4$ & & $8.4 \pm 3.8$ & $8.2 \pm 2.4$ & $9.0 \pm 5.8$ & & \\
\hline Pitavastatin & $11(12 \%)$ & $8(14 \%)$ & $3(9 \%)$ & & $23(13 \%)$ & $21(18 \%)$ & $2(3 \%)$ & & \\
\hline $\begin{array}{l}\text { Pitavastatin dose } \\
\text { (mg) }\end{array}$ & $2.5 \pm 0.9$ & $2.5 \pm 0.9$ & $2.7 \pm 0.9$ & & $2.2 \pm 0.9$ & $2.3 \pm 0.8$ & $2.0 \pm 0$ & & \\
\hline Others & $1(1 \%)$ & $1(\%)$ & $0(0 \%)$ & & $3(2 \%)$ & $2(2 \%)$ & $1(2 \%)$ & & \\
\hline Ezetimibe & $12(13 \%)$ & $9(16 \%)$ & $3(11 \%)$ & 0.53 & $19(11 \%)$ & $13(11 \%)$ & $6(9 \%)$ & 0.80 & 0.55 \\
\hline PCSK-9 inhibitors & $1(1 \%)$ & $0(0 \%)$ & $1(3 \%)$ & 0.37 & $0(0 \%)$ & $0(0 \%)$ & $0(0 \%)$ & NS & 0.34 \\
\hline EPA & $10(11 \%)$ & $8(14 \%)$ & $2(6 \%)$ & 0.32 & $17(9 \%)$ & $11(10 \%)$ & $6(9 \%)$ & $>0.99$ & 0.83 \\
\hline Metformin & $11(12 \%)$ & $2(4 \%)$ & $1(4 \%)$ & $>0.99$ & $9(5 \%)$ & $7(6 \%)$ & $2(3 \%)$ & 0.49 & 0.76 \\
\hline DPP4i & $0(0 \%)$ & $6(11 \%)$ & $5(18 \%)$ & 0.52 & $15(8 \%)$ & $10(9 \%)$ & $5(8 \%)$ & $>0.99$ & 0.39 \\
\hline SGLT2i & $4(4 \%)$ & $3(5 \%)$ & $1(3 \%)$ & $>0.99$ & $6(3 \%)$ & $3(3 \%)$ & $3(5 \%)$ & 0.67 & 0.74 \\
\hline PPI & $36(39 \%)$ & $25(42 \%)$ & $11(32 \%)$ & 0.38 & $67(37 \%)$ & $51(45 \%)$ & $16(24 \%)$ & 0.01 & 0.90 \\
\hline Diuretic & $8(9 \%)$ & $4(7 \%)$ & $4(12 \%)$ & 0.46 & $17(9 \%)$ & $10(9 \%)$ & $7(11 \%)$ & 0.80 & $>0.99$ \\
\hline \multicolumn{10}{|c|}{ 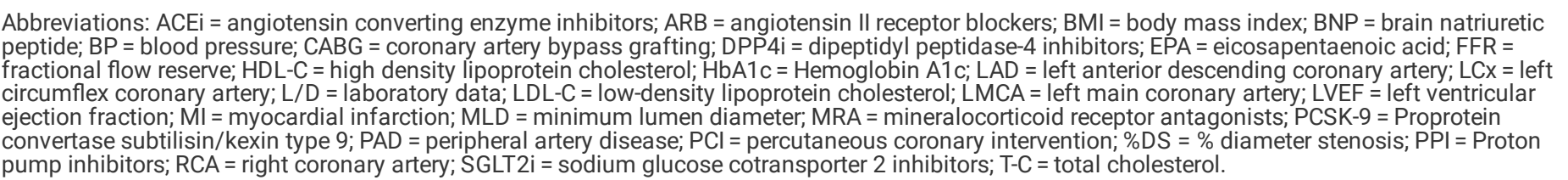 } \\
\hline
\end{tabular}


Among the patients with an FFR of $0.81-0.85$, the lower LDL-C group included significantly more male patients and was more likely to have patients with a lower body mass index and lower systolic blood pressure than the higher LDL-C group. The population that received statins for secondary prevention, that is, patients with a history of prior PCl and coronary artery bypass grafting, were comparably distributed in the lower and higher LDL-C groups. With respect to lipid profiles, there was no significant difference in LDL-C values at FFR measurement between the higher and lower LDL-C groups ( $p=0.06$ ). The mean LDL-C level declined from 100.3 to $74.4 \mathrm{mg} / \mathrm{dL}$ at 1 year after FFR measurement in the lower LDL-C group, while it rose from 114.5 to $120.9 \mathrm{mg} / \mathrm{dL}$ in the higher LDL-C group. Thus, the LDL-C value at 1 year after FFR measurement differed significantly between the higher and lower LDL-C groups ( $<<0.001)$. The statin prescription rates both at FFR measurement and at 1 year after FFR measurement in the lower LDL-C group were significantly higher than those in the higher LDL-C group.

\section{(iii) Patients with FFR $>0.85$}

On the other hand, patients with FFR $>0.85$ showed no significant difference in clinical backgrounds between the higher and lower LDL-C groups, except for the distribution of the target vessel of FFR. FFR measurement was most often performed with the left ascending artery in both groups, and the percentage of measurements with this artery was higher in the lower LDL-C group. Consistent with the findings for patients with an FFR of $0.81-0.85$, the mean LDL-C level declined from 91.9 to $74.1 \mathrm{mg} / \mathrm{dL}$ at 1 year after the FFR measurement in the lower LDL-C group, while it rose from 110.0 to $130.8 \mathrm{mg} / \mathrm{dL}$ in the higher LDL-C group. The statin prescription rates both at FFR measurement and at 1 year after FFR measurement were also significantly higher in the lower LDL-C group than in the higher LDL-C group.

\section{Prognoses}

During the median follow-up period of 663 days, the endpoints were observed in 30 patients (32\%) with an FFR of $0.81-0.85$ and 31 patients (17\%) with FFR > 0.85. Kaplan-Meier analysis showed that the MACCE rate was significantly higher in patients with an FFR of $0.81-0.85$ than in patients with FFR $>0.85$ (logrank, $p=0.003$, Fig. 2). The hazard ratio (HR) for MACCE in patients with an FFR of 0.81-0.85 population was 2.14 (95\% confidence interval [Cl]: $1.28-3.59)$ in comparison with the FFR $>0.85$ population. The details of the endpoint are shown in Table 2 . Unplanned revascularization was the leading cause of MACCEs in each population. Non-fatal myocardial infarction during the observation period occurred in only one patient in each population. In the two patients who developed non-fatal myocardial infarction, the culprit lesion was located outside the target vessel that had been measured FFR in both cases. Univariate Cox regression analysis showed that the FFR value, T-C, LDL-C, and HbA1c levels were associated with MACCE (Table 3). The FFR value and HbA1c levels were independent predictors, even after adjusting for covariates.

Table 2

Details of major adverse cardiovascular and cerebrovascular events

\begin{tabular}{|lll|}
\hline \multicolumn{1}{c}{ events } & FFR 0.81-0.85 & FFR $>0.85$ \\
\cline { 2 - 3 } & $\mathrm{n}=\mathbf{9 3}$ & $\mathrm{n}=\mathbf{1 8 0}$ \\
\hline Total & 30 & 31 \\
\hline Death & 0 & 1 \\
\hline Non-fatal myocardial infarction & 1 & 1 \\
\hline Ischemic stroke & 5 & 2 \\
\hline Heart failure hospitalization & 5 & 7 \\
\hline Unplanned revascularization & 19 & 20 \\
\hline Abbreviations: FFR = fractional flow reserve. & \\
\hline
\end{tabular}


Table 3

Univariate cox analysis in all patients, patients with FFR $0.81-0.85$ and patients with FFR $>0.85$

\begin{tabular}{|c|c|c|c|c|c|c|c|c|c|c|c|c|c|c|c|c|}
\hline \multirow{3}{*}{ Variables } & \multicolumn{6}{|l|}{ All } & \multicolumn{5}{|c|}{ FFR $0.81-0.85$} & \multirow{2}{*}{\multicolumn{5}{|c|}{$\begin{array}{l}\text { FFR }>0.85 \\
\text { Univariate }\end{array}$}} \\
\hline & \multicolumn{3}{|c|}{ Univariate } & \multicolumn{3}{|c|}{ Multivariate } & \multicolumn{3}{|c|}{ Univariate } & \multicolumn{2}{|c|}{ Multivariate } & & & & & \\
\hline & OR & $\begin{array}{l}95 \% \\
\mathrm{Cl}\end{array}$ & $\begin{array}{l}P \\
\text { value }\end{array}$ & OR & $\begin{array}{l}95 \% \\
\mathrm{Cl}\end{array}$ & $\begin{array}{l}P \\
\text { value }\end{array}$ & OR & $\begin{array}{l}95 \% \\
\mathrm{Cl}\end{array}$ & $\begin{array}{l}P \\
\text { value }\end{array}$ & OR & $\begin{array}{l}95 \% \\
\mathrm{Cl}\end{array}$ & $\begin{array}{l}P \\
\text { value }\end{array}$ & OR & $\begin{array}{l}95 \% \\
\mathrm{Cl}\end{array}$ & $\begin{array}{l}\mathrm{P} \\
\text { value }\end{array}$ & OR \\
\hline Age, years & 1.00 & $\begin{array}{l}0.98- \\
1.03\end{array}$ & 0.84 & & & & 1.00 & $\begin{array}{l}0.96- \\
1.03\end{array}$ & 0.83 & & & & 1.01 & $\begin{array}{l}0.97- \\
1.04\end{array}$ & 0.72 & \\
\hline Male & 1.06 & $\begin{array}{l}0.61- \\
1.19\end{array}$ & 0.83 & & & & 0.49 & $\begin{array}{l}0.24- \\
1.04\end{array}$ & 0.06 & & & & 2.83 & $\begin{array}{l}0.99- \\
8.13\end{array}$ & 0.053 & \\
\hline $\mathrm{BMI}, \mathrm{kg} / \mathrm{m}^{2}$ & 1.05 & $\begin{array}{l}0.97- \\
1.13\end{array}$ & 0.26 & & & & 1.06 & $\begin{array}{l}0.95- \\
1.19\end{array}$ & 0.29 & & & & 1.05 & $\begin{array}{l}0.95- \\
1.17\end{array}$ & 0.36 & \\
\hline $\begin{array}{l}\text { Systolic BP, } \\
\mathrm{mmHg}\end{array}$ & 1.01 & $\begin{array}{l}1.00- \\
1.02\end{array}$ & 0.20 & & & & 1.01 & $\begin{array}{l}1.00- \\
1.03\end{array}$ & 0.06 & & & & 1.00 & $\begin{array}{l}0.99- \\
1.01\end{array}$ & 0.92 & \\
\hline Heart rate, bpm & 1.01 & $\begin{array}{l}0.99- \\
1.02\end{array}$ & 0.54 & & & & 1.01 & $\begin{array}{l}0.99- \\
1.03\end{array}$ & 0.30 & & & & 1.00 & $\begin{array}{l}0.97- \\
1.03\end{array}$ & 0.88 & \\
\hline Smoking & 0.65 & $\begin{array}{l}0.35- \\
1.19\end{array}$ & 0.16 & & & & 0.55 & $\begin{array}{l}0.22- \\
1.36\end{array}$ & 0.20 & & & & 0.78 & $\begin{array}{l}0.34- \\
1.79\end{array}$ & 0.56 & \\
\hline Atrial fibrillation & 0.46 & $\begin{array}{l}0.11- \\
1.89\end{array}$ & 0.28 & & & & - & - & - & & & & 1.02 & $\begin{array}{l}0.24- \\
4.40\end{array}$ & 0.98 & \\
\hline Prior MI & 0.74 & $\begin{array}{l}0.35- \\
1.57\end{array}$ & 0.43 & & & & 1.13 & $\begin{array}{l}0.39- \\
3.29\end{array}$ & 0.82 & & & & 0.54 & $\begin{array}{l}0.18- \\
1.60\end{array}$ & 0.26 & \\
\hline Prior PCl & 0.82 & $\begin{array}{l}0.49- \\
1.38\end{array}$ & 0.46 & & & & 0.71 & $\begin{array}{l}0.34- \\
1.50\end{array}$ & 0.37 & & & & 0.95 & $\begin{array}{l}0.46- \\
1.97\end{array}$ & 0.90 & \\
\hline Prior CABG & 0.33 & $\begin{array}{l}0.04- \\
2.53\end{array}$ & 0.28 & & & & 3.04 & $\begin{array}{l}0.41- \\
22.81\end{array}$ & 0.28 & & & & - & - & - & \\
\hline $\begin{array}{l}\text { FFR value (per } \\
0.1 \text { increase) }\end{array}$ & 0.39 & $\begin{array}{l}0.20- \\
0.76\end{array}$ & 0.006 & 0.41 & $\begin{array}{l}0.20- \\
0.82\end{array}$ & 0.01 & 0.87 & $\begin{array}{l}0.07- \\
11.65\end{array}$ & 0.92 & & & & 0.41 & $\begin{array}{l}0.11- \\
1.46\end{array}$ & 0.17 & \\
\hline \multicolumn{17}{|c|}{ L/D and medication at 1 year after FFR measurement } \\
\hline $\begin{array}{l}\text { Hemoglobin, } \\
\text { g/dL }\end{array}$ & 1.03 & $\begin{array}{l}0.90- \\
1.18\end{array}$ & 0.67 & & & & 0.93 & $\begin{array}{l}0.75- \\
1.16\end{array}$ & 0.51 & & & & 1.10 & $\begin{array}{l}0.92- \\
1.32\end{array}$ & 0.27 & \\
\hline $\begin{array}{l}\text { eGFR, } \\
\mathrm{mL} / \mathrm{min} / 1.73 \mathrm{~m} 2\end{array}$ & 0.99 & $\begin{array}{l}0.98- \\
1.00\end{array}$ & 0.16 & & & & 1.01 & $\begin{array}{l}0.99- \\
1.02\end{array}$ & 0.43 & & & & 0.97 & $\begin{array}{l}0.95- \\
0.99\end{array}$ & 0.007 & $0.9 \varepsilon$ \\
\hline $\mathrm{T}-\mathrm{C}, \mathrm{mg} / \mathrm{dL}$ & 1.01 & $\begin{array}{l}1.00- \\
1.01\end{array}$ & 0.013 & & & & 1.01 & $\begin{array}{l}1.00- \\
1.01\end{array}$ & 0.01 & & & & 1.005 & $\begin{array}{l}1.00- \\
1.01\end{array}$ & 0.22 & \\
\hline LDL-C, mg/dL & 1.01 & $\begin{array}{l}1.00- \\
1.01\end{array}$ & 0.017 & 1.01 & $\begin{array}{l}1.00- \\
1.01\end{array}$ & 0.2 & 1.02 & $\begin{array}{l}1.01- \\
1.03\end{array}$ & 0.003 & 1.02 & $\begin{array}{l}1.00- \\
1.03\end{array}$ & 0.008 & 1.01 & $\begin{array}{l}1.00- \\
1.01\end{array}$ & 0.27 & \\
\hline $\mathrm{HDL}-\mathrm{C}, \mathrm{mg} / \mathrm{dL}$ & 0.99 & $\begin{array}{l}0.97- \\
1.01\end{array}$ & 0.34 & & & & 0.99 & $\begin{array}{l}0.96- \\
1.01\end{array}$ & 0.32 & & & & 1.00 & $\begin{array}{l}0.97- \\
1.02\end{array}$ & 0.85 & \\
\hline $\begin{array}{l}\text { Triglyceride, } \\
\mathrm{mg} / \mathrm{dL}\end{array}$ & 1.00 & $\begin{array}{l}1.00- \\
1.002\end{array}$ & 0.29 & & & & 1.00 & $\begin{array}{l}1.00- \\
1.003\end{array}$ & 0.049 & 1.00 & $\begin{array}{l}1.00- \\
1.002\end{array}$ & 0.30 & 1.00 & $\begin{array}{l}0.99- \\
1.00\end{array}$ & 0.29 & \\
\hline HbA1c & 1.45 & $\begin{array}{l}1.11- \\
1.91\end{array}$ & 0.008 & 1.40 & $\begin{array}{l}1.06- \\
1.85\end{array}$ & 0.02 & 0.79 & $\begin{array}{l}0.42- \\
1.47\end{array}$ & 0.45 & & & & 1.85 & $\begin{array}{l}1.37- \\
2.51\end{array}$ & $\begin{array}{l}< \\
0.001\end{array}$ & 1.81 \\
\hline Statin & 0.72 & $\begin{array}{l}0.43- \\
1.21\end{array}$ & 0.22 & & & & 0.58 & $\begin{array}{l}0.28- \\
1.19\end{array}$ & 0.14 & & & & 1.06 & $\begin{array}{l}0.48- \\
2.35\end{array}$ & 0.89 & \\
\hline Ezetimibe & 0.98 & $\begin{array}{l}0.90- \\
1.07\end{array}$ & 0.62 & & & & 1.00 & $\begin{array}{l}0.88- \\
1.13\end{array}$ & 0.98 & & & & 0.97 & $\begin{array}{l}0.86- \\
1.09\end{array}$ & 0.60 & \\
\hline
\end{tabular}

Abbreviations: $\mathrm{FFR}=$ fractional flow reserve; $\mathrm{BMI}=$ body mass index; $\mathrm{BP}=$ blood pressure; $\mathrm{MI}=$ myocardial infarction; $\mathrm{PCl}=$ percutaneous coronary interventio artery bypass grafting; L/D = laboratory data; T-C = total cholesterol; LDL-C = low-density lipoprotein cholesterol; HDL-C = high density lipoprotein cholesterol; $\mathrm{t}$ A1c

In patients with an FFR of $0.81-0.85$, the lower LDL-C group had a significantly lower MACCE rate than the higher LDL-C group (log-rank, $p=0.006$, Fig. $3 A$ ). HR for MACCE in the lower LDL-C group was 0.36 (95\% Cl: 0.17-0.77) compared to the higher LDL-C group. Univariate Cox regression analysis showed that TC, LDL-C, and TG levels were associated with MACCE in this population (Table 3). Multivariate analysis revealed that the LDL-C level was an independent predictor, even after adjusting for covariates. However, there was no significant difference in the MACCE rate between the lower and higher LDL-C groups in patients with FFR $>0.85$ (log-rank, $p=0.84$, Fig. 3B). Among patients with FFR $>0.85$, HR for MACCE in the lower LDL-C group was 0.94 (95\% Cl: $0.43-2.06$ ) compared to the higher LDL-C group. Univariate Cox regression analysis showed that eGFR and HbA1c were predictors of MACCE in this population (Table 3). 
Finally, the $p$-value for the interaction between FFR populations (FFR of $0.81-0.85$ or FFR $>0.85$ ) and LDL-C groups (lower or higher LDL-C) was significant ( $p$ $=0.033$ ).

\section{Discussion}

\section{Principal findings of this study}

Patients with FFR $>0.80$ were successfully further stratified in relation to the risk of MACCE by using an FFR value of 0.85 . The MACCE rate was significantly higher in patients with an FFR of $0.81-0.85$ than in patients with FFR $>0.85$. Uncontrolled LDL-C levels were associated with higher MACCE rates in patients whose PCl was deferred due to an FFR of 0.81-0.85.

\section{High-risk population among patients who were deferred PCI based on FFR}

The FAME trial concluded that FFR-guided PCI had an advantage over angiography-guided PCl in decreasing major adverse cardiac events for up to 2 years after the index procedure. (14) The DEFER trial reported that deferral of PCI for intermediate coronary stenosis based on an FFR $\geq 0.75$ was associated with favorable outcomes during the very long-term follow-up period. (15) These trials established FFR measurement as a common tool for evaluating myocardial ischemia in a cath lab by a feasible and reproducible method with a clear cut-off point. Recently, the IRIS-FFR study analyzed the relationship between the FFR value and the HR for deferred versus revascularized lesions based on a multivariable model predicting major adverse cardiac events, including cardiac death, myocardial infarction, and repeat revascularization. The two lines intersected at an FFR value of 0.79 . (5) An FFR of $0.75-0.80$ is considered as a gray zone for the prognostic performance of PCl. Therefore, we adopted an FFR of 0.80 as the cut-off level for deferral of PCl, and patients with FFR $>0.80$ were analyzed in this trial.

There is limited evidence to stratify the risk of MACCE development in patients with deferred PCl. To clarify the high-risk population among these patients, we performed further stratification of patients with FFR $>0.80$. We adopted a cut-off value of 0.85 with regard for MACCE based on the results from the IRIS-FFR registry. The registry reported that the HR of major adverse cardiac events was 2.48 (95\% Cl:1.47-4.20) in lesions with an FFR of $0.81-0.85$ and 1.60 (95\% $\mathrm{Cl}: 0.91-2.80)$ in lesions with an FFR of 0.86-0.90, in comparison to lesions with an FFR of 0.91-1.0. (5) Our results showing that the MACCE rate was higher in patients with an FFR of $0.81-0.85$ than in patients with an FFR $>0.85$ were consistent with the results from the IRIS-FFR registry. Multivariate analysis also showed that FFR values could be predictor of MACCE in patients whose PCI was deferred on the basis of the FFR value. These results suggested that patients with an FFR of $0.81-0.85$ were a high-risk population among patients whose PCl was deferred on the basis of FFR values, and that these patients should be followed up closely even after deferral of PCl.

\section{Lipid-lowering therapy in patients with deferred PCl on the basis of FFR}

Secondary prevention of cardiovascular events by lipid control in patients who underwent PCI has been established based on evidence from various studies; $(6,10)$ however, treatment guidelines for patients with deferred PCI based on FFR measurement have not been established. In real clinical settings, some patients, especially those without a history of PCl, fall out of monitoring by cardiologists and do not receive sufficient lipid-lowering therapy. Indeed, the present study, which assessed real-world LDL-C management after FFR measurement, revealed that the mean LDL-C level at 1 year after FFR measurement was far from the target LDL-C level for secondary prevention. Of course, at this point, LDL-C level should be controlled under $70 \mathrm{mg} / \mathrm{dL}$ as a target level for secondary prevention in patients at high risk for cardiovascular events. However, in the real clinical settings, the numbers of patients with LDL-C level were very limited. Therefore, we adopted $100 \mathrm{mg} / \mathrm{dL}$ as a cut-off value of LDL-C in this study. In addition, patients did not receive sufficient lipid-lowering therapy regardless of the FFR value in the present study. There was no statistical difference in LDL-C values at 1 year after FFR measurement between patients with an FFR of $0.81-0.85$ and those with FFR $>0.85$.

In the current study, we reported the different prognostic effects of LDL-C management according to the patients' FFR values. An uncontrolled LDL-C level was associated with a higher MACCE rate in patients with an FFR of $0.81-0.85$, who were at a relatively higher risk of MACCE among patients whose PCI was deferred. In contrast, the LDL-C level was not associated with the MACCE rate in patients with FFR $>0.85$, who were at a relatively lower risk of MACCE among patients whose $\mathrm{PCl}$ was deferred. Multivariate Cox regression analysis showed that the LDL-C level was an independent predictor even after adjusting for covariates in patients with an FFR of $0.81-0.85$, but not in patients with FFR $>0.85$. Additionally, the interaction between the FFR population (FFR of $0.81-$ 0.85 or FFR $>0.85$ ) and LDL-C groups (lower or higher LDL-C groups) was statistically significant. These results indicate the importance of LDL-C control, especially in patients with an FFR of $0.81-0.85$. Of note, these results do not indicate that LDL-C management is less important in patients with FFR >0.85. All patients with intermediate coronary stenosis, which were performed FFR measurement, should be received intensive LDL-C management. However, as shown in this study, LDL-C management have been insufficient after FFR measurement in real clinical practice. Therefore, as the first step, the risk stratification presented in this study is an important new perspective.

A close correlation has been reported between achieved LDL-C levels and the changes in the atheroma volume in a prior trial using intravascular ultrasound. (16) According to the GLAGOV randomized trial, an intensive LDL-C-lowering therapy with PCSK9i resulted in a greater decrease in the percent atheroma volume than a normal control. (17) One report described LDL-C control and FFR values for lesions after PCl, and showed that an LDL-C value over 70 mg/dL was associated with an increased FFR value for the target vessel at 18 months after PCl. (18) Since insufficient LDL-C control was associated with a high MACCE rate in patients with an FFR of $0.81-0.85$ but not in those with FFR $>0.85$, insufficient LDL-C could cause an atheroma volume increase or a destabilization of lipid plaque, leading to myocardial ischemia. These results suggest that patients whose PCI was deferred on the basis of an FFR of $0.81-$ 0.85 should be followed up closely and should receive sufficient lipid-lowering therapy.

\section{Study limitations}


This study has several limitations that should be acknowledged. First, this was a retrospective observational study performed in a single center with a relatively small number of patients. Second, the cut-off LDL-C value was $100 \mathrm{mg} / \mathrm{dL}$ in our study, although the guidelines recommended LDL-C $<70 \mathrm{mg} / \mathrm{dL}$ as the target for patients with a very high cardiovascular event risk, such as patients with previous acute coronary syndrome and familial hypercholesterolemia. (10) The reason was mentioned in discussion part. However, the average LDL-C value in the lower LDL-C group was approximately $74 \mathrm{mg} / \mathrm{dL}$ in both FFR groups. Thus, LDL-C control in the lower LDL-C group nearly achieved the strict target under the guideline recommendations. Third, our study excluded patients with multiple intermediate stenoses that required separate FFR measurements because they could not be allocated into subgroup based on FFR value. Therefore, patients with a relatively lower severity of atherosclerosis may have been selected for analysis in the current study. Considering the lower-the-better strategy for LDL-C management, a randomized controlled study to verify the impact of strict LDL-C management in high-risk patients with deferred PCI is warranted.

\section{Conclusions}

Among patients with deferred PCl after FFR measurement, MACCEs were more frequent in patients with FFR $0.81-0.85$ than in those with FFR $>0.85$. Uncontrolled LDL-C levels were associated with higher event rates in patients with deferred PCI due to FFR $0.81-0.85$ but not in those with FFR $>0.85$. These results suggest that even after deferral of PCl, patients with coronary artery stenosis of lower FFR values should receive strict LDL-C-lowering therapy with close monitoring by cardiologists.

\section{Abbreviations And Acronyms}

BNP = brain natriuretic peptide

$C A D=$ coronary artery disease

eGFR = estimated glomerular filtration rate

$F F R=$ fractional flow reserve

HDL-C = high density lipoprotein cholesterol

LDL-C = low-density lipoprotein cholesterol

MACCE = major adverse cardiovascular and cerebrovascular events

$\mathrm{PCl}=$ percutaneous coronary intervention

$\mathrm{T}-\mathrm{C}=$ total cholesterol

$\mathrm{TG}=$ triglyceride

\section{Declarations}

Funding: Not applicable.

Disclosure: There are no conflicts of interest to declare. This manuscript has not been published or presented elsewhere in part or in entirety and is not under consideration by another journal. The study design was approved by the appropriate ethics review board.

Acknowledgements: We thank Editage (www.editage.jp) for English language editing.

\section{References}

1. Pijls NH, De Bruyne B, Peels K et al. Measurement of fractional flow reserve to assess the functional severity of coronary-artery stenoses. $\mathrm{N}$ Engl $\mathrm{J}$ Med 1996;334:1703-8.

2. Bech GJ, De Bruyne B, Pijls NH et al. Fractional flow reserve to determine the appropriateness of angioplasty in moderate coronary stenosis: a randomized trial. Circulation 2001;103:2928-34.

3. De Bruyne B, Fearon WF, Pijls NH et al. Fractional flow reserve-guided PCl for stable coronary artery disease. N Engl J Med 2014;371:1208-17.

4. Barbato E, Toth GG, Johnson NP et al. A Prospective Natural History Study of Coronary Atherosclerosis Using Fractional Flow Reserve. Journal of the American College of Cardiology 2016;68:2247-2255.

5. Ahn JM, Park DW, Shin ES et al. Fractional Flow Reserve and Cardiac Events in Coronary Artery Disease: Data From a Prospective IRIS-FFR Registry (Interventional Cardiology Research Incooperation Society Fractional Flow Reserve). Circulation 2017;135:2241-2251.

6. Knuuti J, Wijns W, Saraste A et al. 2019 ESC Guidelines for the diagnosis and management of chronic coronary syndromes. Eur Heart J 2020;41:407-477.

7. Baigent C, Blackwell L, Emberson J et al. Efficacy and safety of more intensive lowering of LDL cholesterol: a meta-analysis of data from 170,000 participants in 26 randomised trials. Lancet (London, England) 2010;376:1670-81.

8. Boekholdt SM, Hovingh GK, Mora S et al. Very low levels of atherogenic lipoproteins and the risk for cardiovascular events: a meta-analysis of statin trials. Journal of the American College of Cardiology 2014;64:485-94. 
9. Daida H, Teramoto T, Kitagawa Y, Matsushita Y, Sugihara M. The relationship between low-density lipoprotein cholesterol levels and the incidence of cardiovascular disease in high-risk patients treated with pravastatin: main results of the APPROACH-J study. International heart journal 2014;55:39-47.

10. Kinoshita M, Yokote K, Arai H et al. Japan Atherosclerosis Society (JAS) Guidelines for Prevention of Atherosclerotic Cardiovascular Diseases 2017. Journal of atherosclerosis and thrombosis 2018;25:846-984.

11. Yamagishi M, Tamaki N, Akasaka T et al. JCS 2018 Guideline on Diagnosis of Chronic Coronary Heart Diseases. Circulation journal: official journal of the Japanese Circulation Society 2021;85:402-572.

12. Ginsberg HN, Elam MB, Lovato LC et al. Effect of combination lipid therapy in type 2 diabetes mellitus. N Engl J Med 2010;362(17):1563-74

13. Knuuti J, Ballo H, Juarez-Orozco LE et al. The performance of non-invasive tests to rule-in and rule-out significant coronary artery stenosis in patients with stable angina: a meta-analysis focused on post-test disease probability. Eur Heart J 2018;39:3322-3330.

14. van Nunen LX, Zimmermann FM, Tonino PA et al. Fractional flow reserve versus angiography for guidance of PCl in patients with multivessel coronary artery disease (FAME): 5-year follow-up of a randomised controlled trial. Lancet (London, England) 2015;386:1853-60.

15. Zimmermann FM, Ferrara A, Johnson NP et al. Deferral vs. performance of percutaneous coronary intervention of functionally non-significant coronary stenosis: 15-year follow-up of the DEFER trial. Eur Heart J 2015;36:3182-8.

16. Tsujita K, Sugiyama S, Sumida H et al. Impact of Dual Lipid-Lowering Strategy With Ezetimibe and Atorvastatin on Coronary Plaque Regression in Patients With Percutaneous Coronary Intervention: The Multicenter Randomized Controlled PRECISE-IVUS Trial. Journal of the American College of Cardiology 2015;66:495-507.

17. Nicholls SJ, Puri R, Anderson T et al. Effect of Evolocumab on Progression of Coronary Disease in Statin-Treated Patients: The GLAGOV Randomized Clinical Trial. Jama 2016;316:2373-2384.

18. Hashikata T, Tojo T, Muramatsu Y et al. Lower Level of Low Density Lipoprotein Cholesterol is Associated with a Higher Increase in the Fractional Flow Reserve in Patients with Fixed-dose Rosuvastatin. J Atheroscler Thromb 2018;25:233-243

\section{Figures}

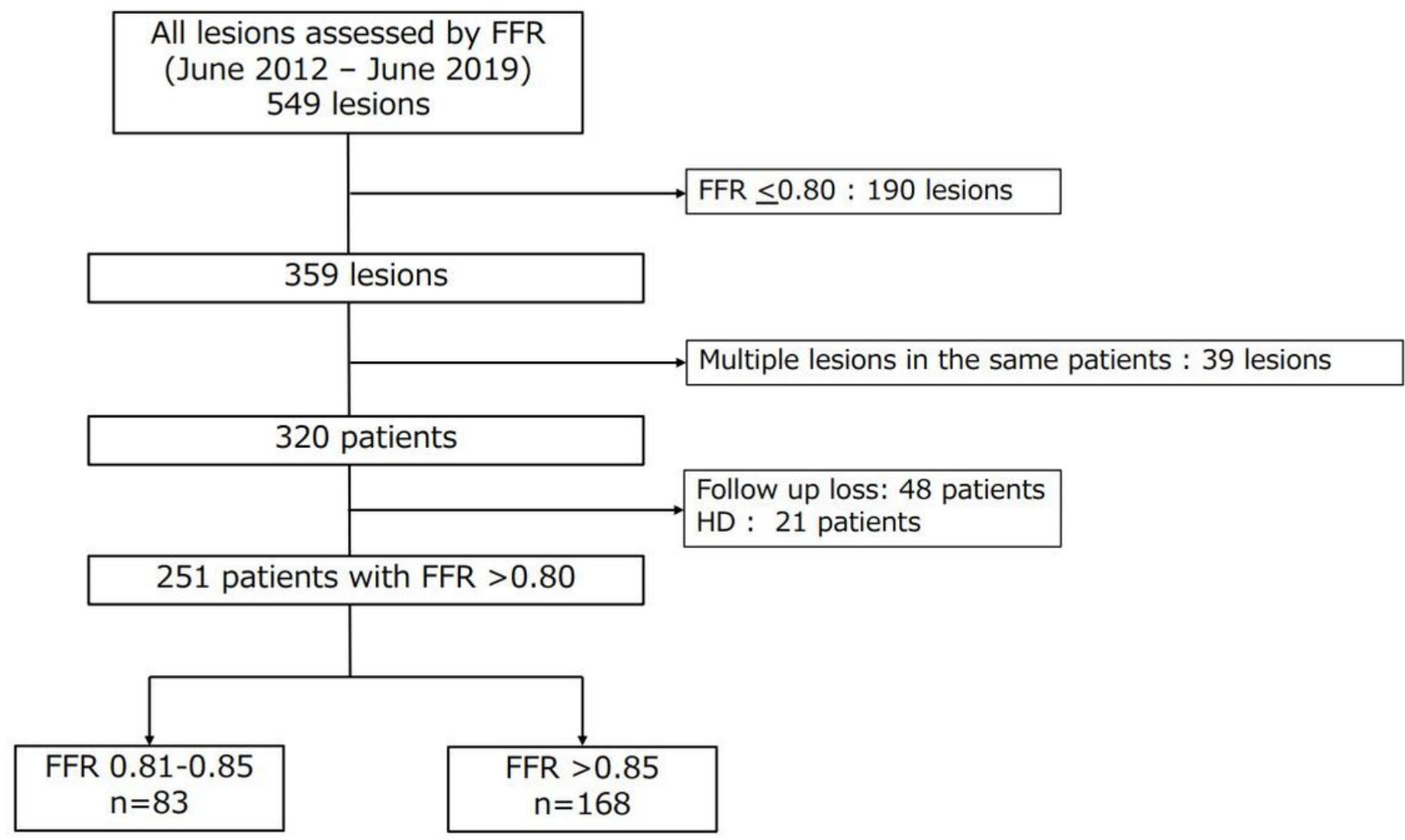

Figure 1

\section{Flow chart of participant recruitment and categorization}

FFR, fractional flow reserve; HD, hemodialysis; LDL-C, low-density lipoprotein cholesterol. 


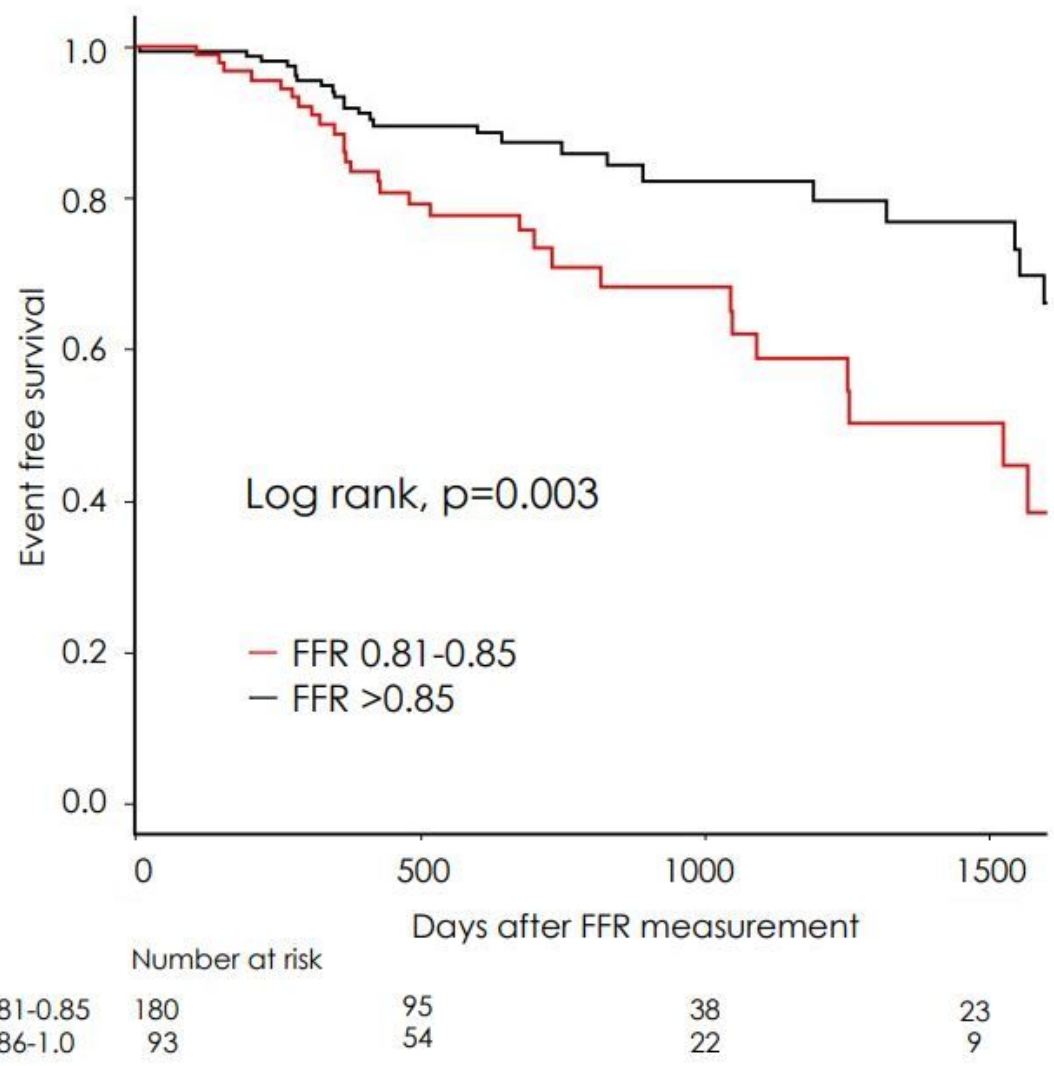

Figure 2

Major adverse cardiovascular and cerebrovascular events in patients with an FFR of $0.81-0.85$ vs. those with FFR $>0.85$

Kaplan-Meier event curves for major adverse cardiovascular and cerebrovascular events in patients with an FFR of 0.81-0.85 and FFR >0.85.

FFR $=$ fractional flow reserve; $L D L-C=$ low-density lipoprotein cholesterol. 

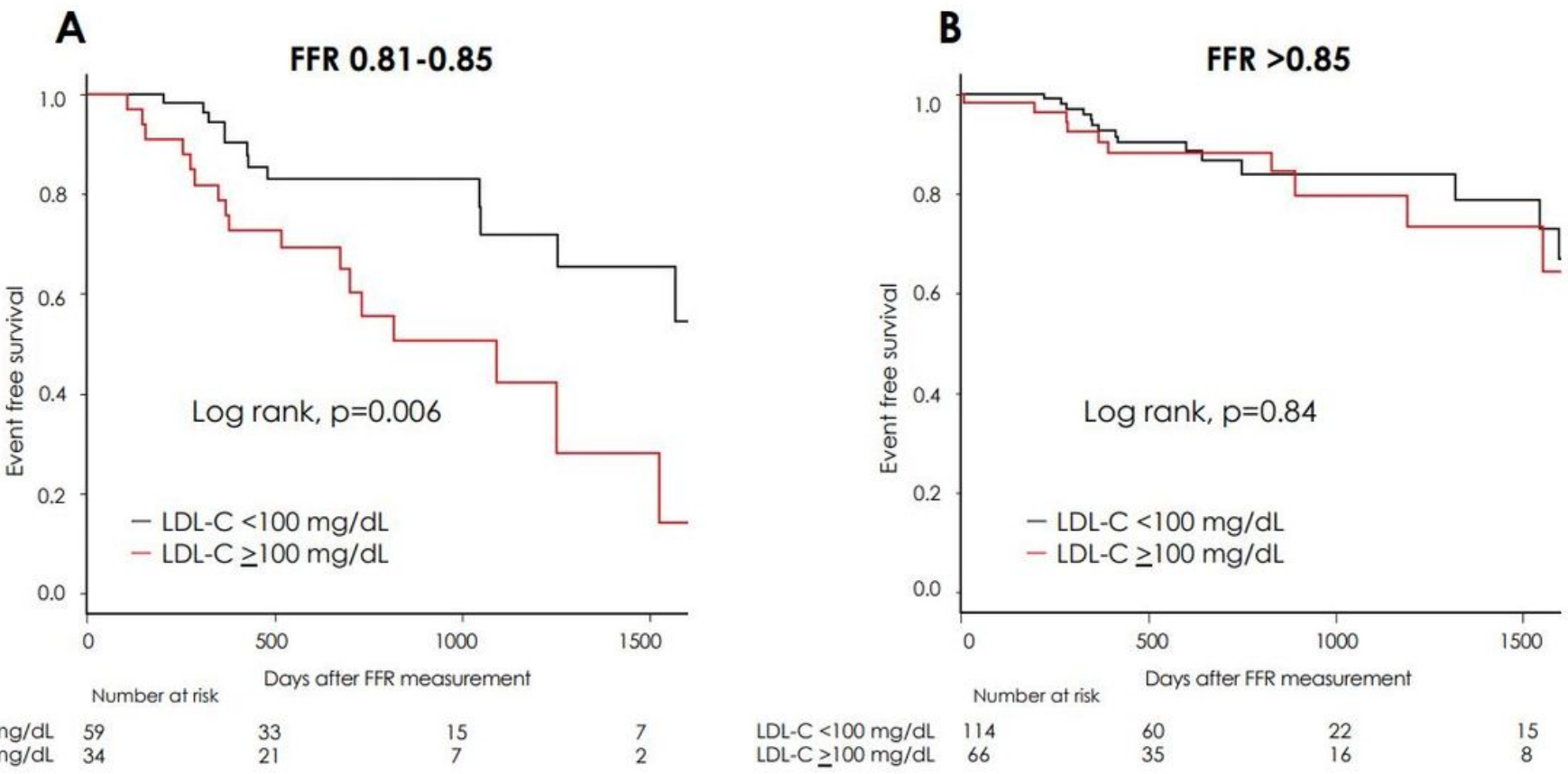

\section{Figure 3}

\section{Major adverse cardiovascular and cerebrovascular events in the higher vs. lower LDL-C groups.}

Kaplan-Meier event curves for major adverse cardiovascular and cerebrovascular events in the higher and lower LDL-C groups with an FFR of $0.81-0.85$ (A) and FFR $>0.85(B)$.

FFR = fractional flow reserve; LDL-C = low-density lipoprotein cholesterol. 